\title{
Throughput Maximization using Spatial Reusability in Multi Hop Wireless Network
}

\author{
Twinkal P. Dudhagawali', Prof. L. K. Gautam², Prof. V. P. Vaidya² \\ ${ }^{1}$ M.E. (I.T.), ${ }^{2}$ Professor \\ 1,2Department of Information Technology, Sipna College of Engineering \& Technology, \\ 1,2Amravati, Maharashtra, India
}

\begin{abstract}
How to cite this paper: Twinkal $\mathrm{P}$. Dudhagawali | Prof. L. K. Gautam | Prof. V. P. Vaidya "Throughput Maximization using Spatial Reusability in Multi Hop Wireless Network" Published in International Journal of Trend in Scientific Research and Development (ijtsrd), ISSN: 24566470, Volume-3 | Issue-3, April 2019, pp.1832-1835, URL: https://www.ijtsrd.c om/papers/ijtsrd23 489.pdf

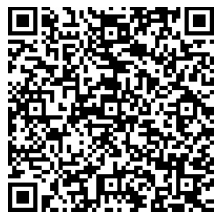
IITSRD23489
\end{abstract}

Copyright (C) 2019 by author(s) and International Journal of Trend in Scientific Research and Development Journal. This is an Open Access article distributed under the terms of the Creative Commons

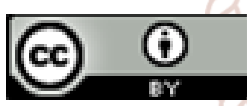
Attribution License (CC BY 4.0) (http://creativecommons.org/license s/by/4.0)

\section{INTRODUCTION}

\section{A. Wireless Sensor Network}

The sensor network includes small and less cost sensing devices together with wireless radio transceiver for examining the environment. It involves the data gathering and transmitting the information to single or multiple sink nodes. The advantage of this network is that it is infrastructure less or external supply for data gathering. The main applications of WSN are wild habitat monitoring, forest fire detection, building safety monitoring, military surveillance and so on[1].

The characteristics of WSN, which have resulted in challenging issues, are as follows:

1. Sensor nodes are exposed to maximum failures.

2. Sensor nodes utilize the transmit message pattern and possess severe bandwidth restraint.

3. Sensor nodes hold scarce quantity of resources.

\section{B. Multihop Wireless Network}

Multi Hop use many wireless hops to communicate information from one sender to receiver. Mobile Multi-hop Ad Hoc Networks are collections of portable nodes joined mutually over a wireless medium. These hubs can move with no limitation and powerfully self compose into subjective and transitory in specially appointed system topologies permitting individuals and gadgets to consistently internetwork in zones with no prior correspondence foundation (e.g., disaster recovery environments).

The most straightforward specially appointed system is a shared system framed by a gathering of stations inside the scope of each other that progressively design themselves to set up a brief single-jump ad-hoc system.

Bluetooth piconet is the example of single-hop ad hoc network. In this ad hoc networks just inter connect devices that are inside the equal communication range. This downside can be overcome by misusing the multi jump specially appointed standard. In this new systems administration standard, the clients gadgets should chivalrously give the functionalities that are typically given by the system foundation. Close hubs can impart specifically by utilizing a solitary jump remote innovation (e.g., Bluetooth, 802.11, and so on.) while gadgets that are not straightforwardly associated trade a couple of information by sending their activity by means of a grouping of middle gadget[2]. 


\section{Spatial Reusability}

It is an efficiency measure that allows use of same spectral link at the same time. Works on wireless routing matrices is done in traditional wireless sensor network. In wireless communication network it is essential to carefully find the high utility route in multi-hop wireless network protocols proposed for multi hop wireless networks. However a basic problem with presented wireless routing protocols is that reduces the number of transmissions to deliver a single packet from a source node to a destination node does not necessarily maximize the end-to-end throughput[3].

\section{Literature Survey}

Atul Adya et al. [4] has proposed A Multi Radio Unification Protocol for IEEE 802.11Wireless Networks. In this, while constructing a multi-hop network using off-the-shelf 802.11 hardware, normally one uses a single ad-hoc network and therefore all nods that participate in that network end up using the same channel. Unfortunately, even when multiple 802.11 NICs are present on the host, each NIC converges on the same physical channel. As a consequence, because of contention only one NIC is used. MUP multiple radios such that frequency (channel) variety is achieved while ensuring that all nodes a reel. Current technique for estimating channel quality is to send probe messages across each channel on a periodic basis and then to compute the roundtrip latency of these messages. For each neighboring node, a node computes its channel quality metric independent of its neighbors' decision. Independent channel selection simplifies the protocol design because no agreement is required between the sender and the receiver on which channel to use.

C. E. Perkins et al.[3] This method allows a group of mobile computers, which may not be near to any base station and can exchange data along altering and random paths, to all computers among their number a (possibly multi-hop) path along which data can be exchanged. In addition, result must remain compatible with operation in cases where a base station is exist. By using the strategy outlined not only will routing be seen to explain the problems with ad-hoc networks, but in addition describe ways to perform such routing functions which traditionally has not been utilized as a protocol level for routing.

J. Broch et al. [4] proposed TORA protocol based on "link reversal " algorithm. It is intended to discover the route on demand provides several routes to the destination establish route quickly and minimize overhead in transmission by localizing algorithmic reaction to the topological changes when possible.

S. Chachulski et al. [5] proposed Opportunistic routing has explains the possible throughput raise and the ExOR method as a way to achieve it. Shrewd routing has a place with a general class of remote calculations that make utilization of multi-client assorted qualities. These procedures utilize gatherings at various hubs to raise remote throughput. They may optimize the alternative of forwarder from those nodes that received a transmission or merge the bits received at different nodes to correct for wireless errors or allow all nodes that overheard a transmission to simultaneously forward the signal acting as a multi-antenna system. The work builds on this foundation but adopts a fundamentally different approach, it combines random network coding with opportunistic routing to address.
D. B. Johnson et al.[8] Dissimilar to routing conventions utilizing distance vector or link state algorithms, utilizes dynamic source directing which adjusts rapidly to routing changes when have development is visit, yet requires practically no overhead amid periods in which host move less. In view of results from a packet level simulation of versatile hosts working in an ad-hoc system, the gathering performs well over an assortment of ecological conditions, for example, host density and development rates. For everything except the most astounding rates of host development recreated, the overhead of the convention is very low, tumbling to only $1 \%$ of aggregate information bundles transmitted for direct development rates in a system of 24 moving hosts.

R. Laufer et al.[9] Author gave an answer for integrating opportunistic routing and various transmission rates. The accessible rate differing qualities forces a few new difficulties to routing, since radio range and conveyance probabilities change with the transmission rate. Given a system topology and a goal, need to discover both a sending set and a transmission rate for each node, with the end goal that their separation to the goal is limited. It represent this as the most brief multi-rate anypath issue. Finding the rate and sending set that mutually advance the separation from a node to a given goal is viewed as an open issue. To understand it, we presented the EATT routing metric and in addition the Shortest Multirate Anypath First (SMAF) calculation and exhibited a verification of its optimality.

\section{System Architecture}

In this routing protocol planned is intended for WSNs in which the sensing nodes are static. Beside the applications running in the WSN require the information collected by the all sensor nodes and have to be send instantly to the sink. All the variables at the each node $\mathrm{j}$ are represented as follows. CFj1 $=$ Value of first cost field of node $\mathrm{j}$

$\mathrm{CFj} 2=$ Value of second cost field of node $\mathrm{j}$

PFj1 = Value of first parent node field of node $j$

$\mathrm{PFj} 2$ = Value of second parent node field of node $\mathrm{j}$

$\mathrm{Nj}=\mathrm{jth}$ node

$\mathrm{REj}=$ Remaining energy of $\mathrm{Nj}$

$\mathrm{Cj}=1$

$\mathrm{REj}=$ Each nodes cost to be added to a path

As shown in Fig. 1 the broadcast tree construction of the routing protocol is having of two stages. In the first stage, the sink node transmits an advertisement message ADV1. Upon reception of ADV1 message, each node of the WSN executes the algorithm given in the procedureBTC-phase1and set its first parent field so that the path to the sink node through it has least cost. Upon completion of the first stage, the sink broadcast a second advertisement message ADV2. Upon receipt of ADV2 message each node of the WSN is start executes the algorithm they are given in the procedure BTCphase 2 and set its second parent field so that the path to the sink node through it has the second least cost. Node j broadcast ADV1and ADV2 has the following parameters.

$A D V 1=(N j, C F j 1, P F j, 1), A D V 2=(N j, C F j, 1)$

BTC-phase 1 describes the how to construct the initial tree which performs its task as follows. At the beginning of first period, each node except the sink node sets its both cost fields to and parent node fields to -1 , but at the starting of the subsequent periods, the each node only sets its both of the cost field to and no change is made to the parent node fields. The sink node sets its both cost fields to 0 and set its 
parent node fields to its own ID. At the beginning of this phase, sink node transmit an ADV1 message to all its neighbours. When a node receives an ADV1 message, it does not broadcast its own ADV message to its neighbour immediately. Following steps are executed before sending the ADV1 message to its neighbors,

1. When a node receives the first ADV1 message, it sets backoff timer.

2. If the first ADV1 message comes from the sink node then node stores to the sink node ID in two parent node field and computes the new cost by adding reciprocal of it's the left over energy to the received cost and stores the new cost in two cost field. If the first ADV1 message comes from the any other node in the network, then the node is compare the new cost with the existing cost stored in the first cost field

3. Upon reception of any further ADV1 message from other neighbors, it is computes the newly cost in the same manner as in the step 2 . If the previous node has already stored the sink node ID in its parent node field, then it will discard the ADV1 message, otherwise, it compare this new cost with the presenting cost stored in its first cost field and updates its cost fields and parent node fields as in step2.

Once the back off timer expires, the node broadcasts ADV1message that contains its own ID, the value stored in the first cost field, and the parent node ID stored in the first Procedure BTC-phase1 begin if (First period).

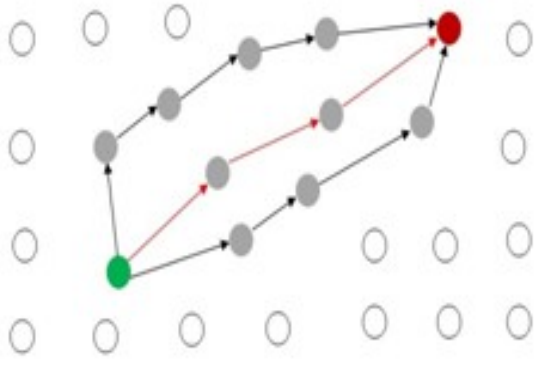

Fig. 1 System Architecture

\section{Procedure: Construction of BTC}

Input: Initial source node $S n$, Destination node $d n$, Group of neighbor nodes nd [], each node $i d$, each node energy eng.

Output: Source to destination path when data received success.

Step 1: User first selects the $S n$ and $d n$

Step 2: choose the packet or file f for data transmission.

Step 3: if (f! =null)

Step 4: read each byte $b$ form fd when reach null

Step 5: send data, initialize cf1,cf2,pf1,pf2

Step 6: while (nd[i] when reach NULL)

Cf1=nd[i].eng

Pf1 $=$ nd [i].id

Cf2 $=$ nsd $[i+1]$.eng

Pf2= nd $[i+1] . i d$

Step 7: if $(\mathrm{cf} 1>\mathrm{cf} 2)$

Cf2=null

Pf2=null

Else

Pf1=pf2

$\mathrm{Cf} 1=\mathrm{cf} 2$;
Pf2=null

Cf2=null

Step 8: end while

Step 9: repeat up to when reach at sink node

\section{Hybrid Cryptography \\ Algorithm: Generate an RSA key pair.}

Input: Required modulus bit length, $\mathrm{k}$.

Output: An RSA key pair ((N,e), d) where $\mathrm{N}$ is the modulus, the product of two primes $(\mathrm{N}=\mathrm{pq})$ not exceeding $\mathrm{k}$ bits in length; e is the public exponent, a number less than and coprime to $(\mathrm{p}-1)(\mathrm{q}-1)$; and $\mathrm{d}$ is the private exponent such that ed $\equiv 1(\bmod (\mathrm{p}-1)(\mathrm{q}-1))$.

Select a value of e from $\{3,5,17,257,65537\}$

repeat

$\mathrm{p} \leftarrow$ gen_prime $(\mathrm{k} / 2)$

until $(p \bmod e) \neq 1$

repeat

$\mathrm{q} \leftarrow$ gen_prime $(\mathrm{k}-\mathrm{k} / 2)$

until $(q \bmod e) \neq 1$

$\mathrm{N} \leftarrow \mathrm{pq}$

$\mathrm{L} \leftarrow(\mathrm{p}-1)(\mathrm{q}-1)$

$\mathrm{d} \leftarrow$ mod_inv $(\mathrm{e}, \mathrm{L})$

Return (N, e, d)

\section{DES Encryption}

Basically we use DES for data encryption single-mindedness. When a device send the data to sink first all the data will be translated with the proposed key and other end telephone will decrypt the same data using same key Compositions of Encryption and Decryption:

Encryption E = eL1 o eL2 .............. o eL16

Decryption D = dL16 o dL15 o ............... dL1

Leader $\mathrm{L}$ is derived from the Password. Here we have 16

rotations. Thus we need 16 Leaders (L1 to L16) from

Password.

L1 = First two minutes of Password.

$\mathrm{L} 2=$ Second two bits of Key

L3 = Third two bits of Password and so on

Steps:

Get Plaintext.

Get Password.

Translate the Characters in binary format.

Derive the Leaders (L1 to L16) from the Password.

Apply the Formula to get the converted and decrypted message.

\section{Mathematical Model}

The proposed system used below mathematical approach. Now here $S$ is the system which including the

$\mathrm{S}=\{\mathrm{S} 1, \mathrm{~S} 2, \mathrm{~S} 3, \mathrm{~S} 4\}$

So, $\mathrm{S}$ is the main set and S1 to S4 all are subset.

$\mathrm{S} 1=\{\mathrm{S} 1 \mathrm{i}\}$ this is the single sink node

$\mathrm{S} 2=\{\mathrm{S} 21, \mathrm{~S} 22 \ldots \ldots \ldots . \mathrm{S} 2 \mathrm{n}\}$ this is the subset of sensor node.

S3 $=$ Inode1, Inode2.......Inoden $\}$ this is the subset of intermediate Running nodes

$\mathrm{S} 4=\{$ Snode1,Snode2.......Snoden $\}$ this is the subset of intermediate sleep nodes

Here $\mathrm{S}$ is proposed approach which handles the linier transmission, the result of transmission as well as receiving Fs $=\{$ Aud, Vid, Img, txt $\}$ these are the file system which will support for data transmission 
The system can handles the linier transmission with minimum 3 hops with sleep scheduling appraoch, the result of transmission as well as receiving

$\mathrm{Fs}=\{\mathrm{dp} 1, \mathrm{dp} 2 \mathrm{dp} 3 . . . . . . . . . . \mathrm{dpn}\}$ these are the file system which will support for data transmission as packets.

\section{Success condition}

If(s1 !=null or network tree load success)

\section{Failure condition}

If ( $\mathrm{s} 1==$ null and network tree loading failure)

$\mathrm{Nd}=\{\mathrm{SN} 1, \mathrm{SN} 2, \mathrm{SN} 3 \ldots \ldots \ldots . . . \mathrm{SNn}\}$

Nd denoted the group of nodes

$\mathrm{Ndi}=\{\mathrm{SNk}, \mathrm{Nd}\}$

Ndi also denoted the group of nodes but all nodes having at least one sink node

\section{Results and Discussion}

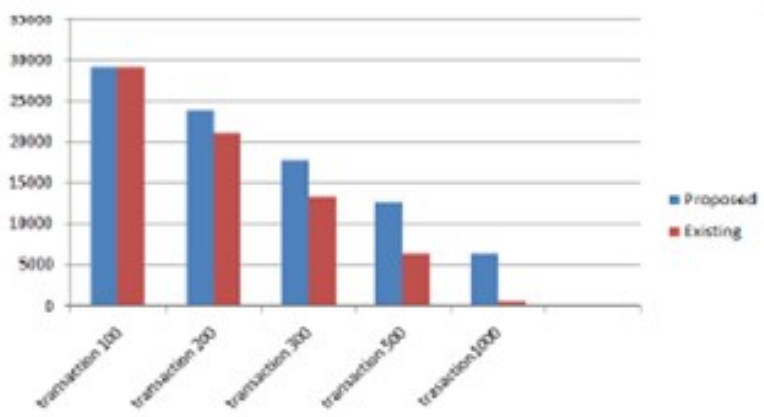

Fig. 2: Energy level after transactions

In given Fig. 2 shows the energy level and $\mathrm{x}$ shows the number of operation of transmission as well as receiving between sink and radar nodes. So we can analyze our proposed system and algorithms are greatly increased the system performance and network live state-owned.

\section{Conclusion}

To overcome the drawbacks of existing system we proposed the Broadcast Tree Construction method which is used to select neighbor node, for data security we used hybrid cryptography, and to improve the network lifetime we proposed the sleep scheduling algorithm. This methods contribute more for better energy efficiency, reduce energy consumption and increase throughput.

\section{References}

[1]. L. Bhasker, "Genetically derived secure cluster-based data aggregation in wireless sensor networks," vol. 8, no. May 2013, pp. 1-7, 2014.

[2]. R. Draves, "Routing in multi-radio, multi-hop wireless mesh networks."

[3]. T. Meng, S. Member, F. Wu, and Z. Yang, "Spatial Reusability-Aware Routing in Multi-Hop Wireless Networks," vol. 65, no. 1, pp. 244-255, 2016.

[4]. A. Adyta, P. Bahl, J. Padhye, A. Wolman, and L. Zhou, "A multi radio unification protocol for IEEE 802.11 wireless networks," in Proc. 1st Int. Conf. Broadband Netw.,2004, pp. 344-354

[5]. C. E. Perkins and P. Bhagwat, "Highly dynamic destination sequenced distance-vector routing (DSDV) for mobile computers," in Proc. Conf. Commun. Archit., Protocols Appl., 1994, pp. 234-244.

[6]. J. Broch, D. A. Maltz, and D. B. Johnson, "A PerformanceComparison of Multi-Hop Wireless Ad Hoc Network Routing Protocols," 1998.

[7]. S. Chachulski, M. Jennings, S. Katti, and D. Katabi, "Trading Structure for Randomness in Wireless Opportunistic Routing," 2007.

[8]. D. B. Johnson and D. A. Maltz, "Dynamic Source Routing in Ad Hoc Wireless Networks."

[9]. R. Laufer and L. Kleinrock, "Multirate Anypath Routing in Wireless Mesh Networks," 2008 